\title{
FT-IR Study of the Molecular Orientation of Rubbed Polyimide
}

\section{Alignment Films}

T.Matsunobe, N.Nagai, R.Kamoto, Y.Nakagawa, and H.Ishida

Toray Research Center, Inc.,

Sonoyama 3-Chome, Otsu, Shiga 520, Japan

\begin{abstract}
Molecular orientations and surface morphology of mechanically rubbed polyimide films have been extensively studied by FT-IR, ellipsometry and atomic force microscope. In-plane and out-of-plane molecular orientations of rubbed polyimide films have been examined by angular dependences of polarized infrared transmission spectra. It has been confirmed that the polyimide chain inclination against the surface plane is induced by the rubbing. It has been shown that mapping of molecular orientation can be obtained by a grazing angle FT-IR microspectroscopy.
\end{abstract}

\section{INTRODUCTION}

It is very important to obtain homogeneous controlled alignment of liquid crystal molecules in the manufacturing process of liquid crystal display (LCD). It is well known that such homogeneous alignment of LC molecules can be achieved by rubbing the polyimide thin film on indium tin oxide (ITO) substrates by nylon or rayon cloth. However, the orientation behavior of the polyimide molecular chain is not clear even at present stage. In the present paper, the thin polyimide films rubbed mechanically at different densities are studied by FT-IR, spectroscopic ellipsometry and atomic force microscope(AFM) in order to clarify ambiguous rubbing mechanism.

\section{EXPERIMENTAL}

\subsection{Sample Preparation}

Polyimide thin films were deposited by a spinning method on the indium tin oxide (ITO) coated silicon and glass substrates as shown in Fig.1. The thickness of polyimide film and ITO were $800 \AA$ and $470 \AA$, respectively. The Si substrate samples were used for the measurement of infrared 
transmission spectra of polyimide films. Polyimide thin films were rubbed at different densities (strong rubbing: $225 \mathrm{~mm}$, medium rubbing: $112 \mathrm{~mm}$, weak rubbing: $36.8 \mathrm{~mm}$ ). The rubbing density(L) is given by the following formula 1$)$ :

$$
\mathrm{L}=\mathrm{NM}(2 \pi \mathrm{rn} / 60 \mathrm{~V}-1)
$$

$\mathrm{N}$ is number of rubbing; $\mathrm{M}$ is contact depth of cloth $(\mathrm{mm}) ; r$ is radius of rotor $(\mathrm{mm}) ; n$ is speed of rotor rotation (rpm); $\mathrm{V}$ is moving speed of stage $(\mathrm{mm} / \mathrm{sec})$.

\subsection{Measurement Procedures}

The IR absorption spectra were measured by Bruker IFS-120HR spectrometer equipped with Mercury Cadmium Tellurium (MCT) detector. For the absorption measurements, 1024 spectral scans were averaged with a $4 \mathrm{~cm}^{-1}$ resolution. The measurements were done under vacuum in order to avoid the influence of vapor.

Fig. 2 shows arrangements for the measurement of angular dependence of infrared transmission spectra. The polarized infrared transmission spectra were measured for the analysis of in-plane molecular orientation by rotating Si substrates with respect to the plane of polarization (Fig.2 a). For the analysis of out-of-plane molecular orientation, the Si substrates were tilted against the direction of polarization (Fig. 2 b). A $10 \mathrm{~mm} \varnothing$ mask was put on the sample surface in order to avoid the effect of the measurement area by tilting.

The IR absorption spectra of micro area
IR measurement

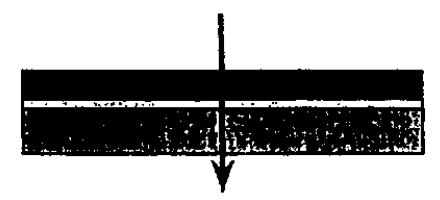

polyimide film

ITO

Si substrate

\section{Measurements of birefringence and AFM}

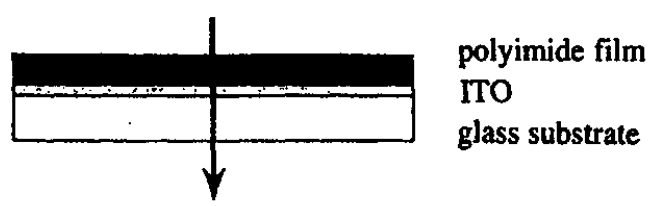

Fig.1 Characterization of molecular orientation of rubbed polyimide films
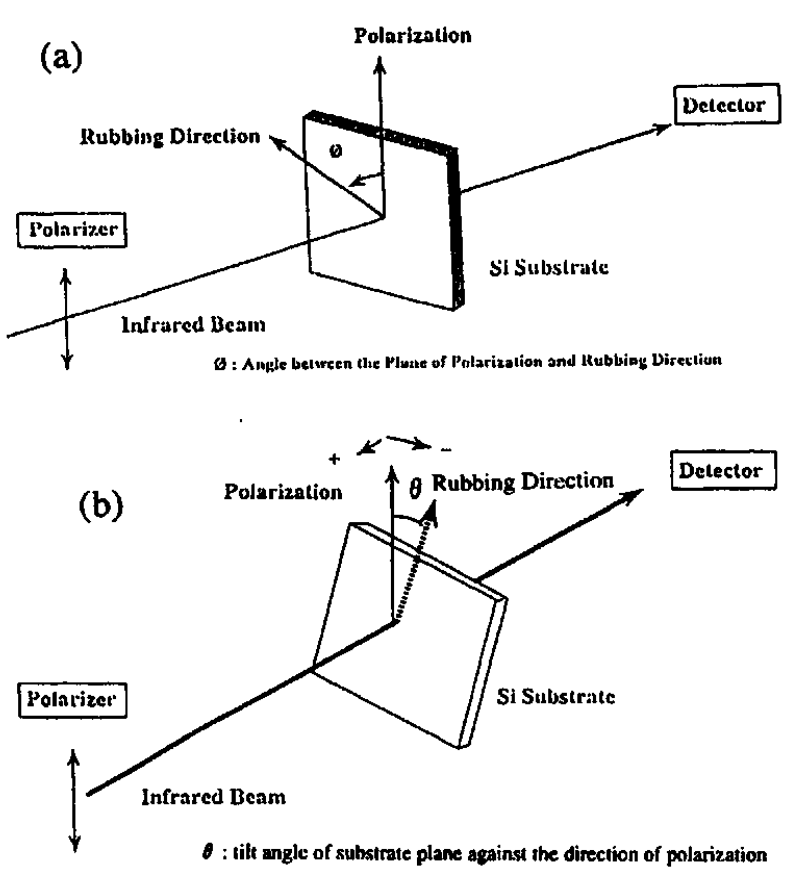

Fig.2 Arrangements used for the measurement of the molecular orientation of rubbed polyimide film by the angular dependence of infrared transmission spectra. (a) analysis of in-plane orientation, (b) analysis of out-of-plane orientation 
of polyimide film were measured by Infrared microscope equipped with a grazing incidence objective (Spectra-Tech IR $\mu$ s), and the thickness of polyimide film were measured by beam profile reflectmetory (Therma Wave OP2000).

The birefringence $(\Delta n)$ of rubbed polyimide films (PI/TTO/glass) were measured by spectroscopic ellipsometry (Nikon NPDM1000 ). The birefringences were measured in near-ultraviolet wavelength region $(420 \mathrm{~nm})$.

Atomic force microscope (AFM, Nano Scope 3 Digital Instruments) was used for the observation of surface morphology of the rubbed polyimide films.

\section{RESULTS and DISCUSSION}

\subsection{Surface Microstructure of Rubbed}

\section{Polyimide Films}

Fig. 3 shows AFM images of rubbed polyimide surface. Microgrooves are observed along the rubbing direction at the intervals from $100 \AA$ to $1000 \AA$. With increasing the rubbing density, the depth and number of grooves increase. The surface roughness estimated from the mean squared roughness $(\mathrm{Rq})$ was found to be proportional to the rubbing density.

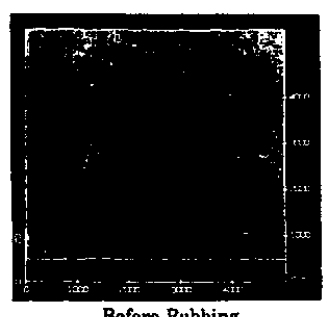

Before Rubbing

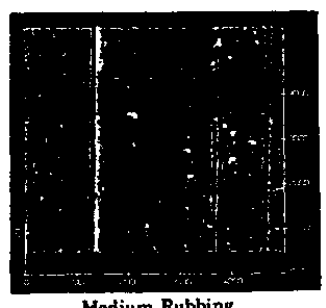

Medium Rubbing
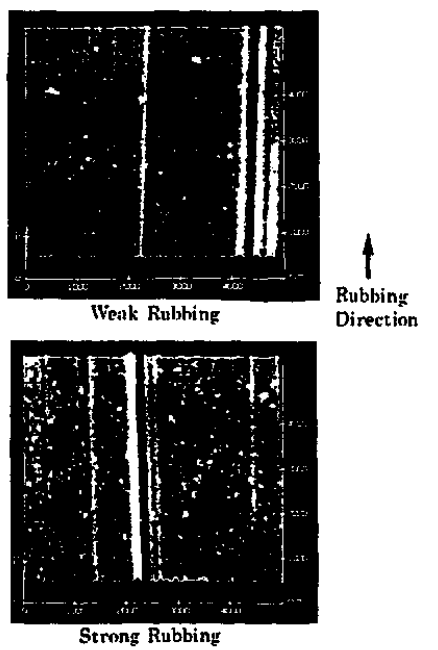

Fig.3 AFM images of rubbed polyimide films

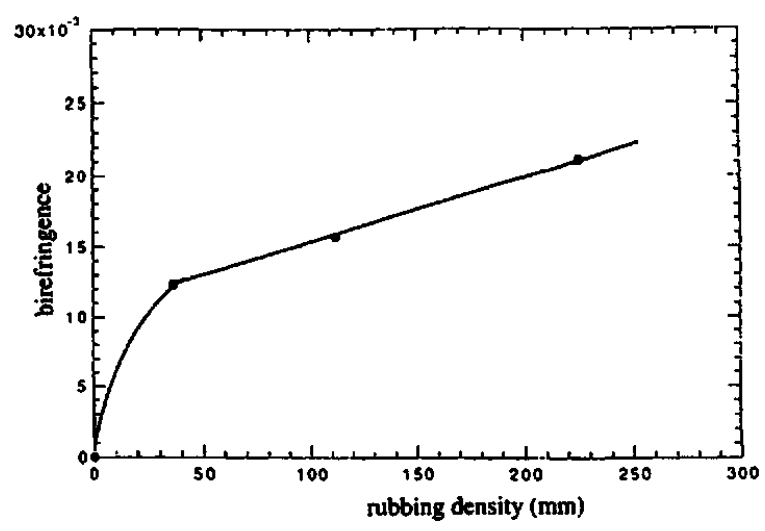

Fig.4 Relationship between the birefringence of rubbed polyimide films and rubbing density

\subsection{Birefringence of Rubbed Polyimide Films}

Fig.4 shows relationship between the rubbing density and the birefringence of the rubbed polyimide films. It is found that the birefringence shows a rapid increase upon the rubbing and a gradual increase above the weak rubbing density. These changes of the birefringence can be explained by the formation of oriented polyimide molecular chains parallel along the rubbing direction, which localize in the surface layer of the film. 


\subsection{Molecular Orientation of Rubbed Polyimide Films Studied by the Angular Dependences of Polarized Infrared Transmission Spectroscopy}

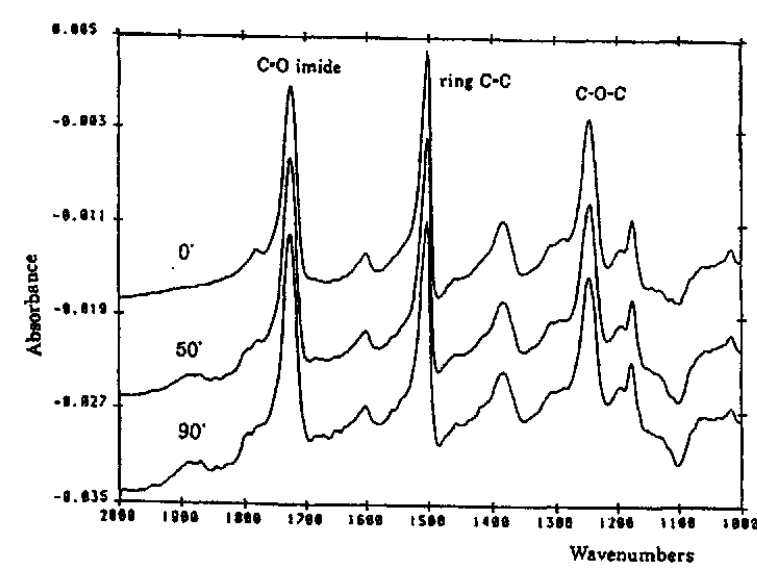

Fig.5 Angular dependence of IR absorption spectra of rubbed polyimide film

Fig.5 shows the polarized infrared transmission spectra of strongly rubbed films on ITO / Si substrates measured at different rotating angles. The stretching vibrations of C-O-C $\left(1245 \mathrm{~cm}^{-1}\right)$ and benzene ring $\mathrm{C}=\mathrm{C}\left(1501 \mathrm{~cm}^{-1}\right)$ have the transition moment parallel to the molecular chain. On the other hand, carbonyl $\mathrm{C}=\mathrm{O}$ stretching vibration in imide functional group has the transition moment almost perpendicular to the molecular chain. The angular dependencies of IR absorption band (C-O-C) are shown in Fig. 6 for the films rubbed at different rubbing densities. It is clearly shown that molecular chains are oriented parallel along the rubbing direction. Fig. 7 shows the relationship between the rubbing density and the IR dichroic ratios of rubbing direction. The molecular orientation estimated from the IR dichroic ratios tends to saturate with increasing the rubbing density. The mechanical rubbing process can not orient homogeneously the overall polyimide film. The surface layer in the polyimide film is induced to orient due to the stress caused by the rubbing. This is the reason why the molecular orientation tends to saturate upon the rubbing density. It has been confirmed that the IR dichroic ratios are proportional to the birefringence of rubbed polyimide films measured by the spectroscopic ellipsometry. This result suggests that the orientation direction of molecular chain

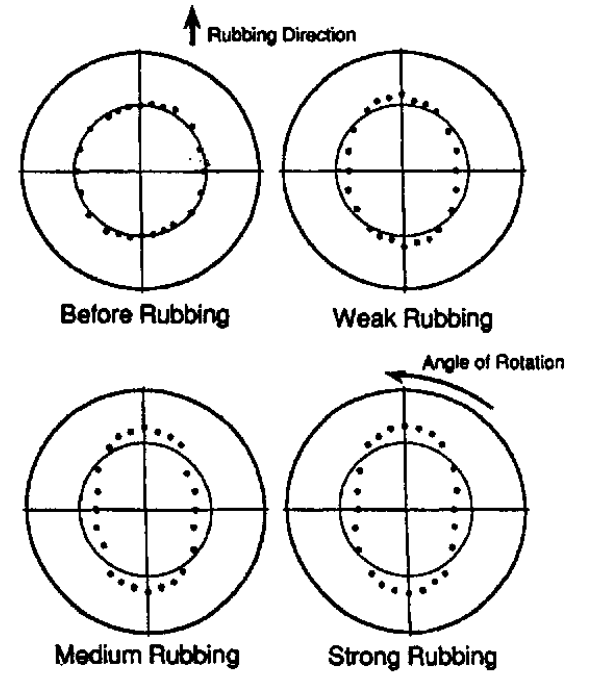

Fig.6 Angular dependences of IR absorption band of rubbed polyimide films (C-O-C at $1245 \mathrm{~cm}^{-1}$ )

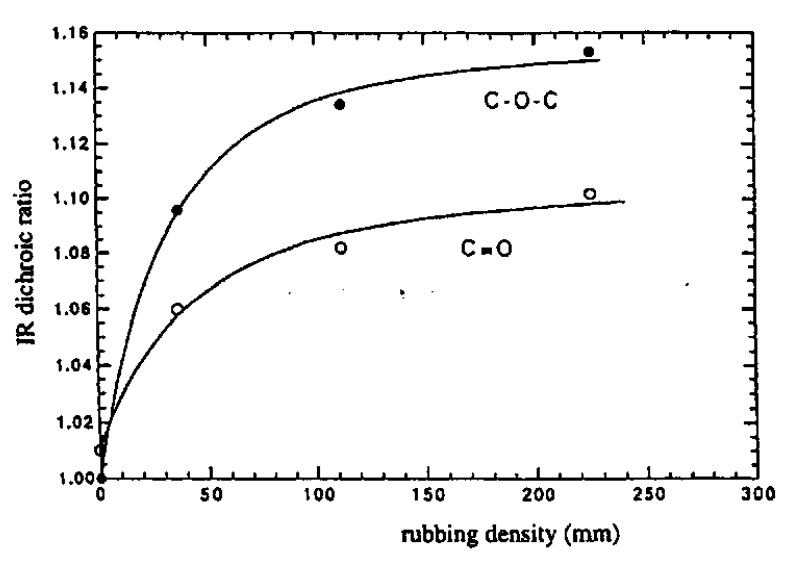

Fig.7 Relationship between the IR dichroic ratios and rubbing density 


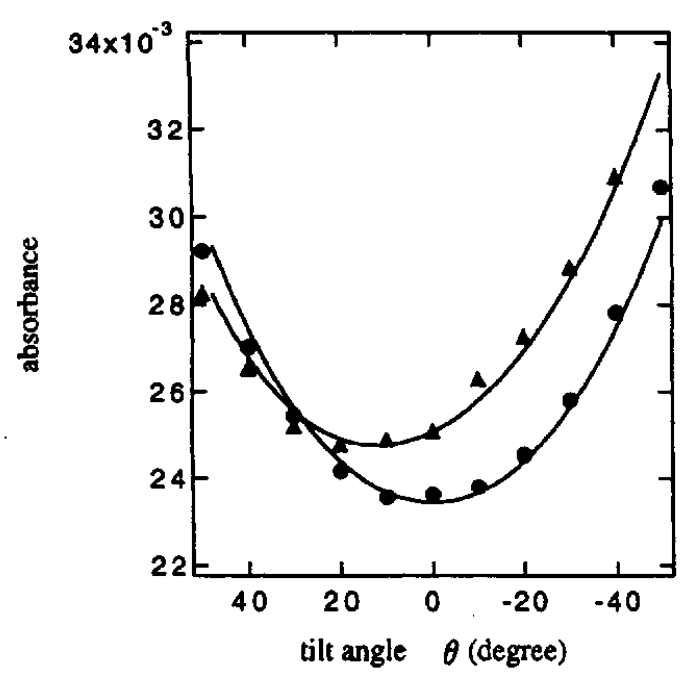

Fig.8 Angular dependence of IR absorption band (C-O-C at $\left.1245 \mathrm{~cm}^{-1}\right)$. The filled circles and triangles are the data points for before rubbing and after strong rubbing, respectively. The solid curves represent the best fitted calculated curves. agrees well with the main optical axis of index ellipsoid.

Recently, it is pointed out that the out-ofplane molecular orientation of polyimide chain plays an important role in the alignment of LC molecules. The out-of-plane molecular orientation can be estimated by the measurement of tilt angle of the molecular chain against the surface plane. As shown in Fig. 2-b, the polarized infrared transmission spectra were measured by inclining the Si substrate against the direction of polarization. Fig. 8 shows the angular dependencies of the IR absorption intensity of C-O-C stretching band for the samples before rubbing and after strong rubbing. The IR band intensity shows symmetrical angular dependence with respect to the vertical axis for the sample before rubbing, while the sample after strong rubbing shows unsymmetric angular dependence. The unsymmetrical dependence strongly suggests the appearance of the tilt angle. The solid curves in Fig. 8 are the curves calculated based on the theoretical formula ${ }^{2)}$ in order to fit the experimental data points. As the result of these fitting

Table .1 Tilt angle of molecular chain, thickness of surface oriented layer and the pretilt angle of LC molecule for the polyimide films rubbed at different densities.

\begin{tabular}{|l|c|c|c|c|}
\hline & $\begin{array}{l}\text { rubbing density } \\
(\mathrm{mm})\end{array}$ & $\begin{array}{l}\text { pretilt angle of } \\
\text { LC molecule } \\
(\text { degree })\end{array}$ & $\begin{array}{l}\text { tilt angle of } \\
\text { molecular chain } \\
(\text { degree })\end{array}$ & $\begin{array}{l}\text { thickness of surface } \\
\text { orientecl layer } \\
(\AA)\end{array}$ \\
\hline $\begin{array}{l}\text { weak } \\
\text { rubbing }\end{array}$ & 36.8 & 4.2 & 20 & 113 \\
\hline $\begin{array}{l}\text { medium } \\
\text { rubbing }\end{array}$ & 112 & 3.8 & 28.6 & 123 \\
\hline $\begin{array}{l}\text { strong } \\
\text { rubbing }\end{array}$ & 225 & 3.52 & 30.5 & 138 \\
\hline
\end{tabular}

calculation, the tilt angle, the refractive index of polyimide film and the thickness of surface oriented layer in the polyimide film are determined.

Table. 1 summarizes the calculated results together with the pretilt angle of LC molecule for ' the rubbed polyimide films. The tilt angle of polyimide chain ranges from $20^{\circ}$ (weak rubbing) to 
$31^{\circ}$ (strong rubbing). The thickness of surface oriented layer ranges from $113 \AA$ to $138 \AA$, which is about $15 \%$ of the entire film thickness. It is shown that the tilt angle of the molecular chain in the rubbed film increases with increasing the rubbing density. On the other hand, the pretilt angle of LC molecule on the rubbed polyimide film shows the opposite change for the rubbing density. These results suggest that the polyimide chain inclination induced by rubbing has not direct correlation with the pretilt angle of LC molecule. Further detailed study of the molecular interaction of LC molecule with the rubbed polyimide surface is now in progress in order to clarify the origin of the pretilt angle.

\subsection{Mapping Analysis of Molecular Orientation of Rubbed Polyimide Films by Grazing Angle FT-IR Microspectroscopy}

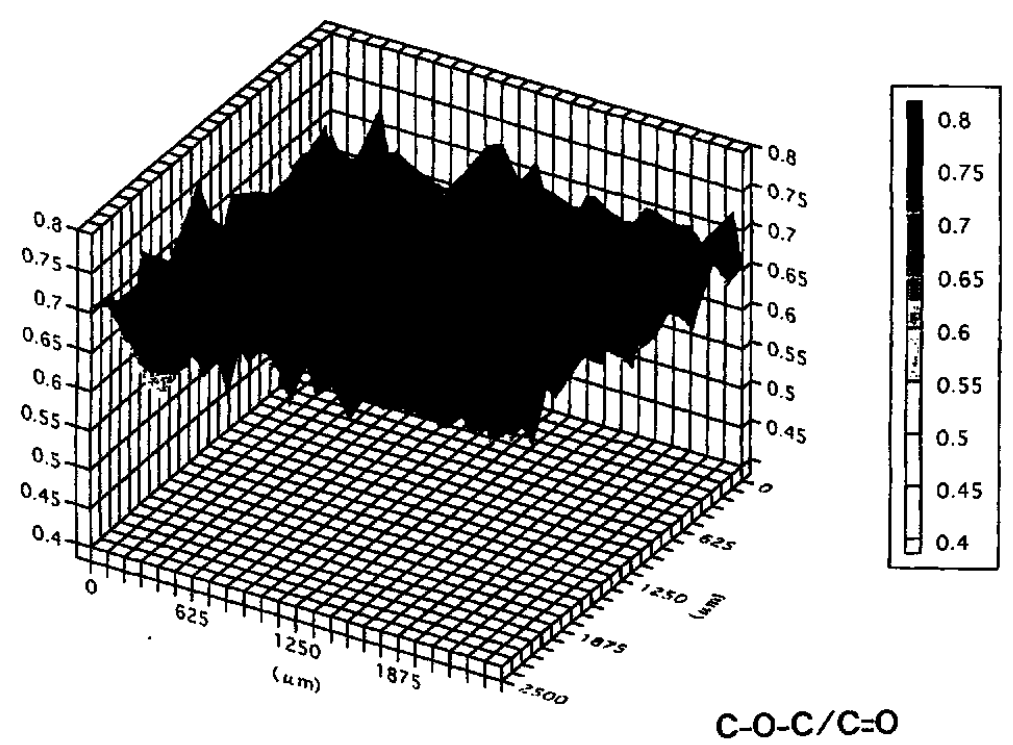

Fig.9 Mapping of the molecular orientation of the strongly rubbed polyimide film

The grazing incidence polarized infrared reflection-absorption spectra (RAS) of a micro-area of the polyimide films can be measured by FT-IR-microspectroscopy (the minimum measurable area : $50 \mu \mathrm{m} \times 50 \mu \mathrm{m})$. In order to obtain the parameter of molecular orientation, RAS spectra were measured with the s-polarized incident beam parallel to the rubbing direction. The RAS spectra were obtained at the good signal-to-noise (S/N) even in $100 \mu \mathrm{mx} 100 \mu \mathrm{m}$ area. Fig.9 shows the mapping analysis of the molecular orientation of the strongly rubbed polyimide films in the $2.5 \mathrm{~mm} \times 2.5 \mathrm{~mm}$ area. This is the first result representing the mapping of the molecular orientation of rubbed polyimide film. It has been found out that the molecular orientation is not homogeneous and shows appreciable fluctuation on the microscopic scale.

\section{REFERENCES}

1) T.Uchida, M.Hirano and H.Sakai : Liq.Cryst.5 (1989) 1127.

2) L.M.Blinov, N.V.Dubinin, L.V.Mikhnen and S.G.Yudin : Thin Solid Films.120 (1984) 161-170. 